\title{
Self-organized Holonic Manufacturing Systems Combining Adaptation and Performance Optimization
}

\author{
José Barbosa $^{1,2,3}$, Paulo Leitão ${ }^{1,4}$, Emmanuel Adam ${ }^{3,5}$, and Damien Trentesaux ${ }^{2,3}$ \\ ${ }^{1}$ Polytechnic Institute of Bragança, Campus Sta Apolónia, Apartado 1134, \\ 5301-857 Bragança, Portugal \\ ${ }^{2}$ Univ. Lille Nord de France, F-59000 Lille, France \\ ${ }^{3}$ UVHC, TEMPO Research Center, F-59313 Valenciennes, France \\ ${ }^{4}$ LIACC - Artificial Intelligence and Computer Science Laboratory, R. Campo Alegre 102, \\ 4169-007 Porto, Portugal \\ ${ }^{5}$ UVHC, LAMIH, F-59313 Valenciennes, France \\ \{jbarbosa, pleitao\} @ipb.pt, \\ \{emmanuel.adam, damien.trentesaux\} @univ-valenciennes.fr\}
}

\begin{abstract}
Traditional manufacturing solutions, based on centralized structures, are ineffective in unpredictable and volatile scenarios. Recent manufacturing paradigms, such as Holonic Manufacturing Systems, handle better these unpredictable situations but aren't able to achieve the performance optimization levels displayed by the classical centralized solutions when the system runs without perturbations. This paper introduces a holonic manufacturing architecture that considers biological insights, namely emergence and selforganization, to achieve adaptation and responsiveness without degrading the performance optimization. For this purpose, self-organization and self-learning mechanisms embedded at micro and macro levels play an important role, as well the design of stabilizers to control the system nervousness in such dynamic and adaptive behaviour.
\end{abstract}

Keywords: Self-organization, Holonic Manufacturing Systems, Distributed Production Control, Bio-inspired engineering.

\section{Introduction}

Traditional manufacturing approaches, built upon centralized and rigid control structures, display near optimal, or even optimal results, but with very restricted assumptions, like the absence of rush orders or resources breakdowns. However, due to the globalization, companies are always pushed to their limits and must keep up with product customization, production volume fluctuation, production cost reduction and internal perturbations, such as machine breakdowns. To face these requirements, the research community have drawn inspiration from several distinct areas of science and propose several manufacturing paradigms, such as Multi-Agent Systems [1], Holonic Manufacturing Systems (HMS) [2], Fractal Factory [3], Reconfigurable Manufacturing Systems (RMS) [4] and Evolvable Production Systems (EPS) [5]. 
Despite the natural differences of these paradigms, they are based on the idea that the controlling layer of the manufacturing system must be decentralized over distributed autonomous entities instead of being monolithically centralized. This decentralization brings some advantages like their inherent capability of adaptation and responsiveness to unexpected perturbations and an increase of the robustness and flexibility, which are aligned with the current requirements imposed to manufacturing companies. However, in spite of all these advantages, the implementation of these paradigms raises some problems, such as the incapability to reach the production performance levels displayed by the traditional centralized systems when the system runs in predictable scenarios. Another problem is related to the organization of these entities to become a coherent, cooperative and collaborative distributed system.

The challenge is to enhance these paradigms with proper mechanisms supporting the combination of their intrinsic characteristics, i.e. flexibility, robustness and adaptation, with the performance optimization provided by traditional centralized structures. For this purpose, the research community can look at the world surrounding, finding answers in nature or biology, and particularly in how species solve their own complex problems and how they self-organize to face complex and dynamic unpredictable situations, as individuals and as a functional working group.

This paper presents the main principles of a holonic manufacturing control architecture that considers biological insights, namely emergence behaviour and selforganization, to combine the performance optimization (usually provided by centralized systems) and adaptation (usually provided by decentralized systems). An important issue in this work is the combination of local and global mechanisms to balance the system micro and macro-levels, allowing the achievement of performance results comparable to those shown by traditional monolithic systems and emergent decentralized paradigms (i.e. combining the best of both worlds).

The rest of the paper is organized as follows: section 2 describes the contribution to value creation and section 3 presents a state of the art in production control paradigms and in biological mechanisms that can inspire the achievement of self-organization and evolution. Section 4 describes the principles of a truly self-organized holonic manufacturing system combining optimization and adaptation, and preliminary results are shown in section 5. Section 6 rounds up the paper with the conclusions.

\section{Contribution to Value Creation}

The emergent manufacturing control paradigms, as referred in the previous section, don't achieve the same levels of performance optimization provided by the traditional hierarchical approaches, despite an increase of performance in situations where the normal functioning condition changes, e.g., due to internal or external perturbations. Bottom line, these paradigms are more adaptable to changing environments but they miss some optimization when the system runs smoothly.

The proposed research work intends to achieve a truly evolvable manufacturing system that combines optimization and adaptation. For this purpose, the proposed approach considers the holonic principles to develop a decentralized manufacturing system that will combine local and global self-organization mechanisms, taking inspiration from biology, which will lead these systems to behave in a more proper way without large fluctuations in performance and with expected higher performance 
levels, even when operating in unstable and turbulent environments. This solution will allow, among others, the increase of the production performance when working in volatile and dynamic environments, characterized by the frequent occurrence of perturbations, through the employment of self-* properties, such as self-adaptation and self-organization. Additionally, other important issues can be expected, such as the decrease of set-up and ramp-up times, the increase of the system flexibility and responsiveness to unexpected scenarios.

\section{State-Of-The-Art in Distributed Manufacturing Control}

This chapter provides an overview of the new emergent manufacturing control paradigms and a brief overview of some mechanisms found in biology that can be used as inspiration to enhance the current emergent manufacturing control paradigms.

\subsection{Current Manufacturing Control Paradigms}

The introduction of the Computer Integrated Manufacturing (CIM) in the 80's forwarded the manufacturing industry into a more reliable and faster functioning, allowing an efficiency improvement, mainly supported by a certain rigidity in its hierarchical topology and process integration. Although this, CIM fails to address, in a proper way, the real manufacturing problems. In fact, in presence of unexpected disturbances, such as the occurrence of resource failures or rush orders, the CIM paradigm may demand long time and effort to re-arrange according to the new variables. To overcome these limitations, new manufacturing control paradigms, based on distributed structures, were reported in the literature, such as RMS, MAS, BMS, HMS and EPS.

RMS is a concept that suggests the rapid change in the factories structure by means of hardware and/or software change. A RMS system should exhibit the following characteristics [4]: modularity, integrability, customization, convertibility and diagnosability. The control layer of such a system can be made by means of MAS, BMS, HMS and EPS.

MAS [1] is simultaneously a paradigm and technology that advocate the creation of a society of decentralized and distributed autonomous and intelligent entities, the agents. Each entity only possesses a partial view of the world surround, requiring the need to cooperate each other to achieve the global objectives.

HMS [2] is a paradigm that translates to the manufacturing world the concepts developed by A. Koestler living organisms and social organizations [6]. A holon, as Koestler devised the term, is an identifiable part of a system that has a unique identity, yet is made up of sub-ordinate parts and in turn is part of a larger whole. A holarchy is a system populated by self-regulating holons, organized in a hierarchical form, being the system goals achieved by the cooperation among holons. An HMS is the encapsulation of the entire manufacturing system into a holarchy, being holons able to represent physical resources and logic entities.

EPS are developed taking inspiration from several fields of science, namely theory of complexity, artificial life or biology, to address dynamic external conditions based on the system reconfiguration principle. The Lego $^{\mathrm{TM}}$ philosophy can be used to explain the system organization as a set of simple, re-configurable and task specific modules, which can be joined in different ways, used to build complex systems. 
Several manufacturing control approaches already use the previous paradigms to improve the system behaviour, namely PROSA (Product, Resource, Order and Staff Reference Architecture) [8], which is a reference holonic architecture that provides a template for the architectural design of manufacturing control systems, and ADACOR (ADAptive holonic COntrol aRchitecture for distributed manufacturing systems) [7] that proposes an adaptive production control approach that balances between two predefined states, intending to be as decentralized as possible and as centralized as necessary, i.e. using a centralized approach when the objective is the optimization, and a heterarchical approach in presence of unexpected events and modifications.

\subsection{Inspiration from Biology in Production Control}

In biology, chemistry, physics or even in nature, entities (e.g., molecules or animals) found ways to maintain their systems running smoothly. Particularly, in the presence of perturbations, it's proved that these systems possess mechanisms that allow them to self-re-organize to adapt to the new conditions and find other points of equilibrium. An example is the food foraging behaviour in ants, where ants are always able to find short routes to food sources by the use of pheromones [9].

In the same way, bees use mechanisms, namely a quantity/quality indicator mechanism known as waggle dance, to organize themselves to work as a coherent group of workers. One last example is the group of birds or fishes that use simple rules of aggregation, limitation of entities and guidance to maintain the system stable.

Shortly, species get organized, by a self-organization concept, which is a process that consists of several interactions, rules and also with spontaneous decisions.

Nature has not only created self-organization and adaptation ways relying in interactions or feedback mechanisms. In fact, Darwin said that in nature, species tend to make changes that over time give origin to new individual behaviours.

The use of bio-inspired mechanisms was applied with success to solve several complex engineering problems, as reported in [9]. In particular, the referred two holonic manufacturing control approaches already consider biological insights; e.g. the PROSA + ANTS approach extends the PROSA principles with inspiration drawn from the ants' food foraging mechanism to forecast future perturbation situations, allowing the system to avoid problems before their occurrence. In spite of the enormous potential of combining the emergent decentralized manufacturing paradigms and insights provided by different areas of science, the answers to some questions are missing, namely the achievement of adaptability without degrade the system performance; the maintaining of the stability and the robustness in very dynamic, adaptive and reactive systems; the combination of local and global selforganization mechanisms; control of the emergence in unpredictable systems and how to identify the not desired emergent behaviours; the implementation of selflearning strategies to support self-organization and self-adaptation?

The answer to these and other questions will let manufacturing systems to perform better and achieve, in a concrete way, higher levels of system performance.

\section{Research Contribution and Innovation}

The current manufacturing problem can be depicted using the Fig. 1, where the curve $h i(t)$ represents the states over time of a traditional hierarchical system. In this 
behaviour, the system displays an optimal performance when all runs according to the plan, but presents a significant decrease in its performance (or even entering in an extreme situation of complete stoppage) when unexpected perturbations deviates the system from the normal plan. The behaviour of decentralized approaches, he(t), shows a considerable performance improvement in situations where the system must adapt due to plan deviations (e.g., the resource malfunction). On the other hand, these systems don't achieve the performance levels displayed by hierarchical approaches for normal conditions. Nevertheless, the overtime operational performance, given by the integration of the curves, can be greater in the heterarchical approach if the system is running in very unpredictable scenarios.

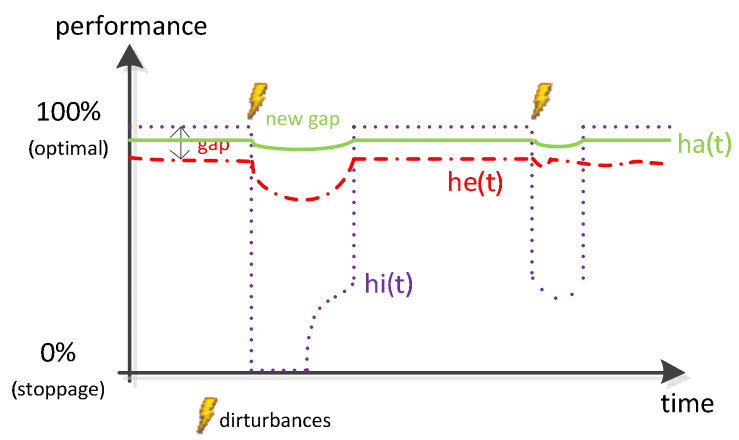

$$
\begin{gathered}
\text { Current: } \\
\text { hi(t) } \geq \text { he }(t) \\
\int \mathrm{he}(t) \geq \int \mathrm{hi}(t) \\
\text { Challenge: } \\
\text { hi(t) } \geq \operatorname{ha}(t) \geq \operatorname{he}(t) \\
\int \mathrm{ha}(t) \geq \int \operatorname{he}(t) \geq \int \mathrm{hi}(t)
\end{gathered}
$$

Fig. 1. Hierarchical and heterarchical performance levels

So, a dichotomy appears when systems perform better than the others according to different situations. At this stage, the research question that can be thrown is "What if a system has the best of both worlds?". What happen if a system reacts better to unpredictable changes than the hierarchical approach and is able to reach its levels of performance in normal conditions?

The research work described in this paper aims to provide insights that combine local and global bio-inspired mechanisms allowing reaching high performance levels with a consistent degree of operational work while is taken into consideration the unification of system optimization and adaptation. Briefly, the desired system, $h a(t)$, will have performance levels greater than those displayed by heterarchical systems, while achieving an overtime performance levels that surpasses the $h i(t)$ and $h e(t)$ performance behaviours.

For this purpose, the ADACOR holonic architecture will be used as the foundation platform, since it has proven to be effective to handle adaptive production control systems [10], considering some biological inspired concepts, such as division of labour and self-organization. Although the performance displayed in the demonstrators [10], the ADACOR approach don't completely achieve a truly evolvable and adaptive system, since it only considers two pre-defined states, i.e., stationary and transient, being the first one related to a more hierarchical configuration and the other to a more flat heterarchical functioning.

The evolution of the ADACOR architecture, as illustrated in Fig. 2, enhanced with more powerful biological insights, will overcome this limitation by allowing the system to evolve in a natural way, e.g., like swarms do in nature. If one observes the 
evolution and behaviour of species it can be seen that, over time, species make small internal changes to cope external conditionings, allowing individuals to acquire, lose or improve new behaviours as needed. As a group, biological societies tend to overcome difficult situations by changing relations or groups associations.

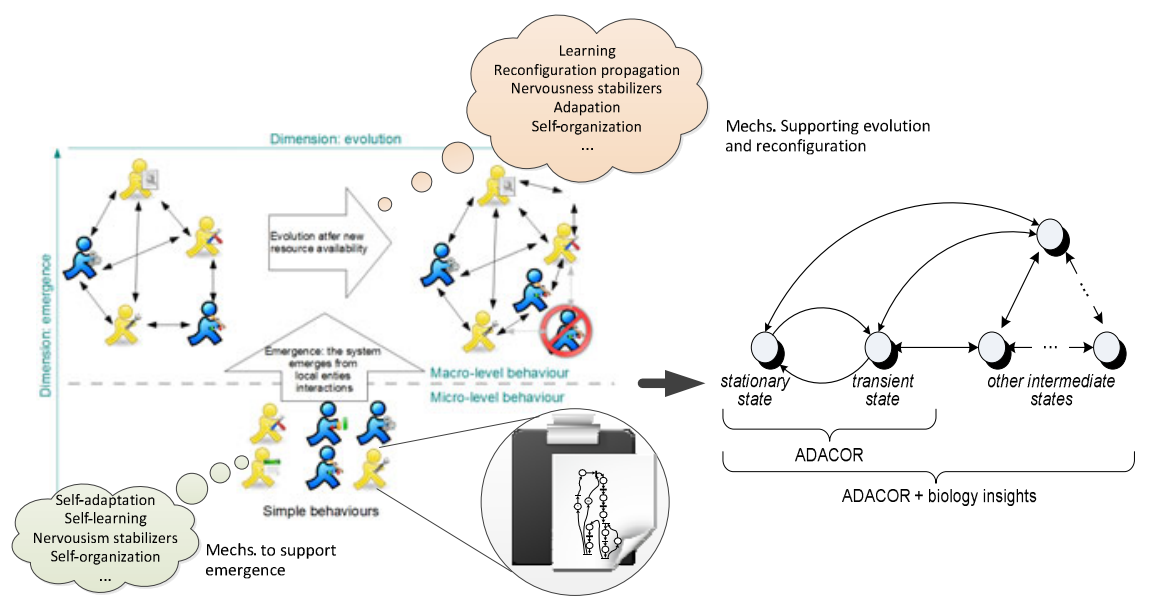

Fig. 2. Emergence and evolution in distributed systems

In this way, individual entities, the holons, will embed several important mechanisms, such as self-adaptation, self-organization and self-learning, that will allow reaching very dynamic and adaptive behaviours. The interactions among holons, each one providing different skills and knowledge, give origin to an emergent global behaviour that is greater than the simple sum of all individual skills. This is represented in Fig. 2 by the emergence dimension.

The second dimension defined in the proposed model is related to the system evolution to address the changing conditions imposed by the environment, namely the occurrence of perturbations or the appearance of new information. This evolution towards the system re-configuration is supported in two distinct manners:

- The micro-level self-organization that is related to the self-organization of the behaviour of individual holons, provoking the emergence of a new global behaviour, and in this way a system adaptation. For this purpose, individual holons use the embedded learning and discover mechanisms to detect new opportunities to evolve and the proper way to re-configure its behaviour.

- The macro-level self-organization, related to the re-organization of the interactions among the holons, provokes a new global behaviour based on a new society of holons. For this purpose, the embedded learning and discovering mechanisms are used to select the proper partners to cooperate according to the new system conditions.

This dynamic and self-organized behaviour may turn the system stability into chaos if entities are allowed to change intentions without pre-defined rules or boundaries. As consequence, the proposed holonic architecture defines a sort of stabilizers, like bumpers in cars, to control the system nervousness, i.e. pushing the system to its 
limits but under control. The stabilizers are embedded in individual holons, exhibiting a profile similar to Proportional, Integrative and Derivative controllers, being also considered at macro-level, e.g., using pheromone-like techniques.

Another key point in the proposed approach is the myopia problem, which appears in distributed systems when the entities only possess a partial view of the world surrounding. For this purpose, the combination of local and global knowledge assumes a crucial importance and drives the achievement of global optimization of the production performance. Pheromone-like mechanisms, as those found in the ants' behaviour, can provide the system to become less myopic since the local information emerges into global information using indirect communication.

\section{Discussion of Results and Critical View}

The described ideas were applied to an experimental routing problem in a dynamic assembly system, composed by 11 conveyors distributed in two levels, being the first one composed by 9 conveyors and the lower level by 2 conveyors; these two levels are connected by two lifters (for more details please consult [11]). Shortly, products arrive at the system with a process plan and must take routing decisions in a proper way to its fulfilment leaving the system afterwards.

The pallets, conveyors and machines belonging in the system were modelled as holons, according to the proposed architecture. Two different mechanisms were embedded in the pallet holons to support the routing decision-making [12]: centralized (optimal approach) and distributed (self-organized approach).

The first decision approach is based on the extraction of the T-invariants proprieties of the cell's Petri Net model and is based on the work of [12]. Basically, this method extracts all the possible routes and when facing a decisional node the pallet holon knows exactly the best path to take. The second approach uses a selforganization method based on the stigmergy concept. During the system operation, the pallet holons, when reaching a target resource, update the information stored at the decisional nodes, as ants deposit pheromones, weighted by the time spent to reach it. Over time, several solutions appear but only the best combination of reinforcement /evaporation of the pheromones in the decisional nodes emerge to be the best path.

The preliminary results show that the self-organizing mechanism embedded in the holons behaviour brings great advantages in terms of system adaptation without losing optimization. In fact, this approach, using very simple methods in this case study implementation, achieves values of performance close to those displayed by the centralized, optimal approach, but provides better responsiveness and adaptation to changes in the system configuration, e.g. due to breakdown of conveyors or traffic jam. In these unexpected situations, the self-organized holonic approach is less penalized than the centralized approach that requires the off-line re-calculation of the optimal plan, which is not feasible to achieve fast and real-time decisions. In this situation the proposed self-organized holonic solution provides an easy and efficient way to quickly adapt to new system configuration.

\section{Conclusions and Further Work}

Emergent manufacturing paradigms proved to be suitable approaches to support the development of distributed control solutions addressing the current requirements 
imposed to manufacturing companies. Although their promising perspectives, it is clear that some aspects need to be addressed and improved to accomplish the grail of manufacturing control. Questions like how to achieve adaptability without degrading the system performance, how to balance local and global perspectives and how to maintain the stability in dynamic and adaptive systems, are far from be answered.

This paper describes a holonic manufacturing architecture that considers biological insights, namely emergence behaviour and self-organization, to achieve adaptation and responsiveness without degrading the performance optimization. The preliminary simulation tests have shown that biology can provide very good insights to overcome the mentioned questions, allowing reaching robustness and adaptability gains without degrading the system performance.

Future work will pass by improving the self-organization models at micro and macro levels and their dependency with self-learning capabilities and the nervousness aspects of individual holons.

\section{References}

1. Ferber, J.: Multi-Agent System: An Introduction to Distributed Artificial Intelligence. Addison-Wesley Professional (1999)

2. Deen, S.: Agent-Based Manufacturing: Advances in the Holonic Approach. Springer, Heidelberg (2003)

3. Warnecke, H.J.: The Fractal Company. Springer, Heidelberg (1993)

4. Mehrabi, M.G., Ulsoy, G., Koren, Y.: Reconfigurable Manufacturing Systems: Key to Future Manufacturing. Journal of Intelligent Manufacturing 11(4), 403-419 (2000)

5. Ribeiro, L., Barata, J., Cândido, G., Onori, M.: Evolvable Production Systems: An Integrated View on Recent Developments. In: Huang, G.Q., Mak, K.L., Maropoulos, P.G. (eds.) DET2009 Proceedings. Advances in Intelligent and Soft Computing, vol. 66, pp. 841-854. Springer, Heidelberg (2010)

6. Koestler, A.: The Ghost in the Machine. Arkana Books (1969)

7. Leitão, P., Restivo, F.: ADACOR: a Holonic Architecture for Agile and Adaptive Manufacturing Control. Computers in Industry 57(2), 121-130 (2006)

8. Brussel, H., Wyns, J., Valckenaers, P., Bongaerts, L.: Reference Architecture for Holonic Manufacturing Systems: PROSA. Computers in Industry 37(3), 255-274 (1998)

9. Barbosa, J., Leitão, P., Trentesaux, D.: Bio-inspired Multi-Agent Systems for Reconfigurable Manufacturing Systems, Engineering Applications of Artificial Intelligence, doi:10.1016/j.engappai.2011.09.025 (2012)

10. Leitão, P., Restivo, F.: Implementation of a Holonic Control System in a Flexible Manufacturing System. IEEE Transactions on Systems, Man and Cybernetics - Part C 38(5), 699-709 (2008)

11. Barbosa, J., Leitão, P.: Modelling and simulating self-organizing agent-based manufacturing systems. In: 36th Annual Conference on IEEE Industrial Electronics Society, pp. 2702-2707 (2010)

12. Leitão, P., Alves, J., Mendes, J.M., Colombo, A.W.: Energy Aware Knowledge Extraction from Petri Nets Supporting Decision-making in Service-oriented Automation. In: Proc. of the IEEE Int'l Symposium on Industrial Electronics, pp. 3521-3526 (2010) 